$17^{\text {th }}$ European Symposium on Computer Aided Process Engineering - ESCAPE17

V. Plesu and P.S. Agachi (Editors)

(C) 2007 Elsevier B.V. All rights reserved.

\title{
Integrated product and process design approach for rationalization of food products
}

\author{
Cristhian Almeida-Rivera ${ }^{\mathrm{a}}$, Puneet Jain ${ }^{\mathrm{a}, \mathrm{b}}$, Solke Bruin ${ }^{\mathrm{b}}$, Peter Bongers ${ }^{\mathrm{a}}$ \\ ${ }^{a}$ Process and Supply Chain Design - Unilever Food and Health Research Institute, \\ Olivier van Noortlaan 120, PO Box 114, 3130 AC Vlaardingen, The Netherlands, \\ cristhian.almeida-rivera@Unilever.com; peter.bongers@Unilever.com \\ ${ }^{b}$ Chair "Product-driven process technology", Eindhoven University of Technology, \\ POBox 513, Helix, STW 1.47, 5600 MB Eindhoven, The Netherlands, s.bruin@tue.nl
}

\begin{abstract}
To quantify rationalization of ingredients and to realize its opportunity during process synthesis, in this contribution we propose an integrated product and process design approach for foods. According to this methodology, once the product concept and geographical market are decided, product attributes that drive consumer liking are identified and quantified. The product attributes are either of sensorial or analytical nature. The identification and quantification of this relationship is obtained by means of data mining techniques. Relevant sensorial and analytical attributes are used, afterwards, to create alternatives for possible microstructures and ingredients. Subsequently, the ingredients are used as inputs for process synthesis to find optimal process to deliver required analytical/sensorial attributes through desired microstructures. The ingredients are related to analytical and sensorial attributes through mathematical process and ingredients models, respectively. We integrate this methodology with a modified Douglas' methodology for food products. By putting together mathematical relationships between consumer liking attributes, product attributes and ingredients and processes, a quantitative rationalization can be achieved. An oil-in-water mayonnaise-like emulsion was used as case study.
\end{abstract}




\section{Introduction}

Unilever is one of the world's largest consumer goods companies, where same products are often made in different plants using different processes to satisfy different consumer likings around the world. On top of this variability, the majority of the products have large number of ingredients, which may show different properties depending upon the sources, season, origin, etc. All these differences in processes and raw materials can have various adverse effects: (a) lack of consistency in the quality of different batches of the products; (b) loss of production if the product does not meet the required quality standards; (c) difficulty in regularly changing the process conditions to accommodate for differences; (d) requirement of large storage space and more manpower, and; (e) increased possibility of making mistakes due to the large number of different kinds of ingredients.

These problems can be overcome or reduced by rationalizing the ingredients in the products. Rationalization is referred in this context as the task of reducing the number of ingredients in a product and optimizing the level of ingredients to deliver the right product quality. In view of the increasing importance of integrating product and process design, we propose in this contribution a way of first quantifying the product development activity and link the consumer likings to process design for foods. With this new methodology we wish to achieve selection of ingredients and processes simultaneously and exploit the opportunity of developing novel micro-structures through innovative processing.

\section{Problem background}

The ultimate goal of rationalization in food products is delivering the right product quality. Thus, there is a need to develop the right product that will satisfy consumers' needs and expectations. As quality of food products depends upon selecting the right micro-structure and the right process for getting this micro-structure, the rationalization of food products involves two aspects,

- Product design, where we identify consumer needs and translate them into new and improved products.

- Process design, where we find the most optimal process to convert inputs (pre-specified ingredients and formulation) into outputs (pre-specified microstructure and tangible, measurable product characteristics (e.g. viscosity and droplet size distribution)).

Currently, these two activities are carried out sequentially. Product is only rationalized during product development, where its microstructure, formulation and product characteristics are fixed. Having defined only qualitative relationships between consumer needs and product characteristics, process synthesis is then used to find the most optimal process to convert inputs (pre- 
specified ingredients and formulation) into outputs (pre-specified microstructure and product characteristics).

In contrast to bulk chemicals, structured products (Fig. 1) are characterized not only by the level of each ingredient (i.e. composition, purity, physical state, temperature, pressure, etc.), but also by the relative spatial arrangement of each ingredient. All these features are responsible for the exclusive attributes of structured products (e.g. creaminess of an icecream, spoonability of a mayonnaise, etc).
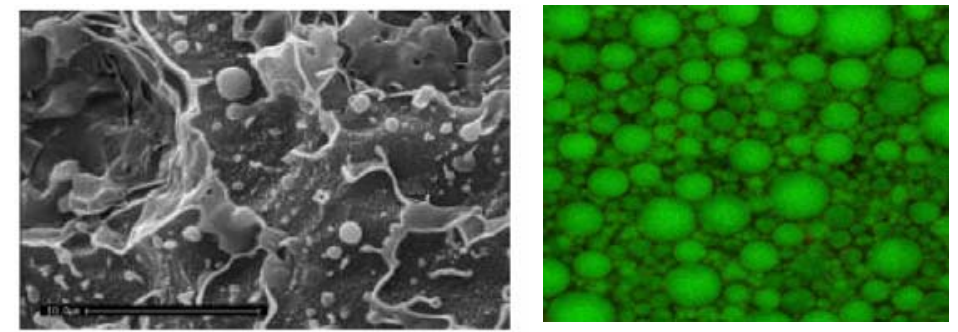

Figure 1. Left: lamellar structured hair conditioner [1]; right: confocal microscopy photographs for a full-fat $\mathrm{O} / \mathrm{W}$ emulsion stained with Nile Blue.

For food products, moreover, quality is a strong function of not only physical properties but also sensorial attributes (e.g. texture, taste, aroma and flavour).

\section{Proposed methodology}

Despite the considerable effort invested in the development of product and process design methodologies, there are some inherent shortcomings of both types of approaches when it comes to their applicability to food (structured) products.

Although product design approaches can reveal which technical parameters are important to achieve the desired quality of products, these parameters are related to customer requirements only qualitatively. The choice of ingredients, product formulation and micro-structure are fixed at this level. Although the levels and choice of ingredients can also be affected by processes, most of the times the development methodologies do not take into account any further possibility of rationalization by processes considerations. In product development methodologies the qualitative relationship between technical parameters and consumer likings does not provide any information on the changes needed in food recipe to achieve certain consumer likings.

Current process design methodologies focus on finding the best process flowsheet (from an economics perspective) to accomplish a given task. In conventional approaches for process synthesis, inputs and outputs are commonly pre-specified. For food products, however, there should be flexibility 
to incorporate changes in inputs with changing outputs and the sensorial attributes and microbiological status of the product should be considered as outputs in the design problem. Moreover, as current methodologies include the micro-structure as an input in process synthesis, we miss the opportunity to explore different micro-structures which may possibly deliver the required product quality.

The big challenge is to incorporate consumer needs in the process design approach. In essence, this proposed methodology keeps focus on customers and their changing needs, and quantitatively predict parameters needed to achieve product quality to satisfy these needs. Additionally, it also looks at the processes and ingredients, aiming at finding the optimal solution to achieve these parameters. As depicted in Fig. 2 the proposed methodology looks at the rationalization problem by linking consumer likings to processes and ingredients. It tries to bring together all three in a quantitative manner, thus overcoming the shortcomings of product development methodologies (e.g. Quality Functional Deployment [2]) and process synthesis [3-5].

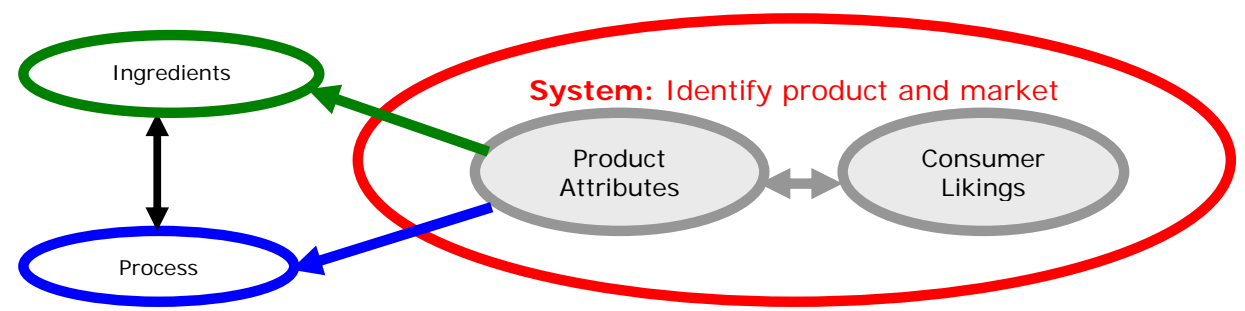

Figure 2. Schematic representation of the rationalization problem.

This methodology involves the following five steps,

Step 1: Define the system by identifying the products and markets to be looked into; carry out the necessary economic and branding benefits.

Step 2: Identify the product attributes most important for consumer liking for the market under consideration. This step identifies the needs of the consumers.

Step 3: Identify the analytical and sensory parameters necessary to define the consumer liking parameters identified at step 2. These parameters are quantitatively related to product attributes important for consumer liking.

Step 4: Relate these analytical and sensory parameters to ingredients either through process and/or ingredient models. Both sensory and analytical parameters are affected by ingredients. To simply the prediction of sensory parameters, the effects of process and ingredients on sensory and analytical parameters are de-coupled in ingredient and process models.

- Step 4a - Ingredient model: ingredients are chosen based on trends of sensorial parameters; these ingredients are related to sensorial parameters through ingredient models. 
- Step $4 b$ - Process model: this methodology can be integrated with the adapted Douglas' methodology for foods [6].

At this step we identify any limitations on ingredients and process conditions that could stem from regulatory, control, environmental, health and hygiene issues.

Step 5: Put all the relationships in mathematical form to find any optimal formulation and/or process conditions. The parameters included in this methodology cover layers related to consumers, processes and ingredients.

\section{Case study}

An oil-in-water mayonnaise-like emulsion was used as case study. After having defined the product concept and market location, consumer liking scores were obtained using a central location technique for a wide range of comparable products. Subsequently sensorial attributes were defined by a trained panel using a descriptive analysis test. These liking scores for each consumer segment were related quantitatively to sensorial and analytical attributes by means of Partial Least Square (PLS) regression method (Table 1) and neural networks (NN) technique. NN technique was chosen over PLS due to its ability to predict some of the non-linear relationships between sensorial and analytical attributes and consumer liking scores (Table 2).

Table 1. Statistical PLS models for prediction of consumer overall likings of a given population segment. Nomenclature: RegCo: regression coefficient; A: analytical parameter; S: sensorial parameter

\begin{tabular}{lcccccc} 
& \multicolumn{2}{c}{ Sensorial } & \multicolumn{2}{c}{ Analytical } & \multicolumn{2}{c}{ Sensorial + Analytical } \\
\cline { 2 - 7 } & & RegCo & \multicolumn{3}{c}{ RegCo } & RegCo \\
& S1 & 0.28 & A1 & 0.29 & A2 & 0.18 \\
& S2 & -0.25 & A2 & 0.16 & S5 & 0.12 \\
& S3 & -0.21 & A3 & 0.21 & A1 & 0.19 \\
& S4 & 0.11 & A4 & -0.12 & A5 & -0.09 \\
& S5 & 0.12 & & & A6 & 0.12 \\
\hline $\mathbf{R}^{2}$-Pred. & & $\mathbf{8 2}$ & & $\mathbf{8 1}$ & & $\mathbf{8 4}$ \\
$\mathbf{R}^{2}$-fit & & $\mathbf{9 1}$ & & $\mathbf{9 0}$ & & $\mathbf{8 8}$ \\
\hline
\end{tabular}

The analytical and sensory attributes were then related to ingredients and processes quantitatively through first principle and/or black-box mathematical models. These models (Fig. 3) were used to define trends of analytical and sensorial attributes and subsequently to develop alternatives for microstructures. As a spin-off of this methodology, we identified white spots existing in the fundamental understanding of product development and process design. This was the case of predictive models which relate ingredients and process to 
analytical parameters of food products. Such white spots are suggested as focus of future research.

Table 2. Comparison of Statistical PLS and NN models for prediction of consumer likings of a given population segment. Nomenclature: RMSE: root mean square error

\begin{tabular}{lcc}
\hline & PLS (RMSE) & NN (RMSE) \\
\hline Sensorial & 0.19 & 0.17 \\
Analytical & 0.19 & 0.11 \\
Sensorial + Analytical & 0.18 & 0.14 \\
\hline
\end{tabular}

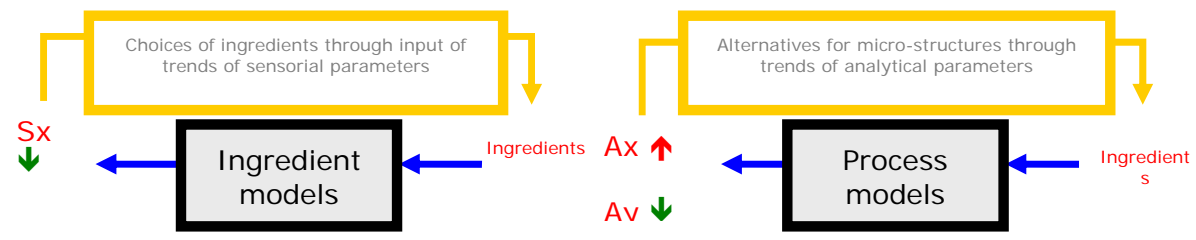

Figure 3. Ingredient and process models for the case study

\section{Conclusions and future work}

In this methodology, a quantitative relationship has been developed between consumer liking parameters and product characteristics. The trends of sensorial and analytical parameters have been then used to choose ingredients and different possible micro-structures which satisfy the trends of these parameters. As for the development of the ingredient and process models, a sound mathematical understanding of processes and ingredients' interactions is required, the proposed approach can also identify any white spots in the knowledge domain. Due to inability of PLS technique to account for the nonlinear relationships between sensorial/analytical parameters and consumer likings, it is recommended to further exploit the power of advanced data mining techniques (e.g. NN).

\section{References}

1. M. Hill, AIChE Journal, 50(2000), 1656

2. S. Bruin, Lecture notes 'Product design', Technical University of Eindhoven, 2004

3. J.M. Douglas, Conceptual design of chemical process, McGraw Hill, New York, 1988.

4. J.J. Siirola, Computers and Chemical Engineering, 20 (1996), S1637

5. J.J. Siirola, Industrial applications of chemical process synthesis, Advances in Chemical Engineering, 23, J.L. Anderson (Ed.), 1996

6. M. vander Stappen, NPT, 12(2005), 22 